\title{
Concurrent B-cell Follicular and Refractory CD30+ Large Cell Transformation of Mycosis Fungoides Responsive to Rituximab and Brentuximab Vedotin
}

Oluchi C. Ukaegbu, Meghan E. Caldwell, and Nishitha M. Reddy*

Departments of Medicine, Division of Hematology/Oncology, Vanderbilt University Medical Center, Nashville TN, USA

*Corresponding author: Nishitha M. Reddy, , Departments of Medicine, 3927 The Vanderbilt Clinic, Vanderbilt-Ingram Cancer Center, Nashville, TN- 37232, USA, Tel: (615) 936-0381; Fax: (615) 936-1812; E-mail: nishitha.reddy@vanderbilt.edu

Received date: Feb 10, 2015, Accepted date: Mar 16, 2015, Publication date: Mar 19, 2015

Copyright: (c) 2015 Ukaegbu OC, et al. This is an open-access article distributed under the terms of the Creative Commons Attribution License, which permits unrestricted use, distribution, and reproduction in any medium, provided the original author and source are credited.

\section{Introduction}

Mycosis fungoides (MF) is a cutaneous T-cell lymphoma that typically follows an indolent course. Transformation of MF to large cell lymphoma, though rare, when occurs, carries a poor prognosis. Development of second malignancies in patients with lymphoma is a previously recognized entity. The coexistence of MF and B-cell malignancy in the same patient however, is uncommon. Herein, we describe a rare presentation of follicular B-cell lymphoma in a patient with known cutaneous T-cell lymphoma. Patient was treated with monoclonal antibody therapy and attained a complete remission of both lymphoma phenotypes.

Keywords: Mycoses fungoides; Brentuximab vedotin; Large cell transformation; Follicular lymphoma

\section{Case Report}

A 51 year-old gentleman presented with an erythematous eruption on face, trunk and extremities in 2001, for which he had a skin biopsy that revealed an atypical lymphoid infiltrate. He was intermittently treated with pimecrolimus with some response. A repeat skin biopsy of a scalp lesion showed a dense infiltrate of $\mathrm{CD} 3+, \mathrm{CD} 4+$ infiltrating irregular ceribriform lymphocytes with a sparse population of CD8+ cells and scattered CD30+ cells that was consistent with involvement by folliculotropic MF with increased numbers of large cells. Patient was initially treated with topical clobetasol, Targretin and psoralen with ultraviolet A (PUVA). PUVA was administered between April 2004 and June 2007 with decreasing frequency. He experienced disease recurrence shortly after being weaned off PUVA with minimal response to carmustine solution. In December 2007, patient was treated with 22 fractions of XRT to a total of 3960 cGy to the right cheek. This resulted in complete resolution of the lesion. In September 2008, he developed a new right nasolabial lesion that initially improved with topical pimecrolimus with progression that was treated with cryotherapy and triamcinolone injection and reinitiated on monthly PUVA therapy. Patient had disease stabilization with this regimen until March, 2011, when he presented with a new right flank lesion for which he underwent radiation therapy, 22 fractions (3960 cGy) with complete resolution.

In November 2011, patient reported a new tender lesion on the right posterior scalp measuring $2.7 \mathrm{~cm}$ and a $2 \mathrm{~cm}$ right forearm lesion. Punch biopsy of both the skin lesions revealed aggregates of atypical lymphoid cells with enlarged nuclei and scattered mitotic figures consistent with cutaneous lymphoma with changes suggestive of large- cell transformation. The patient underwent CT scans of the chest, abdomen and pelvis that showed mesenteric and retroperitoneal adenopathy measuring $1.6 \times 2 \mathrm{~cm}$. Biopsy of these lymph nodes revealed follicular centers containing small lymphocytes with irregular nuclear borders and cleaved cell morphology and immunophenotyping CD20+, CD10+, BCL-6+, BCL-2+, CD5-, CD23- characteristic of follicular lymphoma (FL) grade 1-2 with no evidence of MF. No treatment was initiated for the FL due to low disease burden. The two areas of cutaneous T-cell lymphoma were treated with 22 fractions (3600 cGy) of radiation and topical Targretin with improvement. In August 2012, the patient developed a cutaneous right forearm and lateral thigh lesion each treated with 18 fractions (3600 cGy) of local radiation therapy followed by an additional 9 fractions (1620 cGy) of radiation to the forearm. The forearm lesion proved refractory to radiation therapy and Targretin leading to administration of vorinostat (histone deacetylase inhibitor). Meanwhile, the patient received 20 fractions (3600 cGy) of radiation to a new cutaneous lesion with complete resolution. However, the lesion on the right forearm continued to progress and therapy was switched to romidepsin. Following one cycle of therapy, the lesion on the right forearm continued to further increase in size. Biopsy of the right forearm lesion in June 2013 was consistent with an aggressive tumor notable for a Ki-67 of $70-80 \%$ and was positive for CD30 expression by immunohistochemistry similar to prior biopsies. A repeat CT scan at this time revealed progression of the retroperitoneal and mesenteric adenopathy that previously was consistent with FL. The patient was initiated on therapy with rituximab and Brentuximab Vedotin (BV). Rituximab was administered at a dose of $375 \mathrm{mg} / \mathrm{m}^{2}$, weekly for four treatments followed by every other month for eight months. BV was administered at a dose of $1.8 \mathrm{mg} / \mathrm{kg}$ once every two weeks. He underwent 6 cycles of therapy with BV (cycles 4-6 were dose reduced to $1.2 \mathrm{mg} / \mathrm{m}^{2}$ due to grade 2 neuropathy) with complete resolution of the forearm lesion. A post treatment CT scan revealed complete resolution of lymphadenopathy (Figures 1 and 2). The patient remained in a complete remission for 14 months when he presented with a testicular mass. Pathology of tissue following orchiectomy showed large abnormal lymphocytes with clumped chromatin, nuclear membrane irregularities with scan cytoplasm. Immunohistochemical studies show these abnormal lymphocytes positive for CD3, CD4 and negative for CD8, CD5. CD30 was diffusely positive. This was consistent with large cell transformation of MF. Prior to initiating systemic therapy, patient presented with recurrent skin nodules. $\mathrm{He}$ was initiated on therapy with BV, cyclophosphamide, Adriamycin and prednisone for six cycles again achieving a complete remission. 
Citation: Ukaegbu OC, Caldwell ME, Reddy NM (2015) Concurrent B-cell Follicular and Refractory CD30+ Large Cell Transformation of Mycosis Fungoides Responsive to Rituximab and Brentuximab Vedotin. J Blood Disorders Transf 6: 259. doi:10.4172/2155-9864.1000259

Page 2 of 3
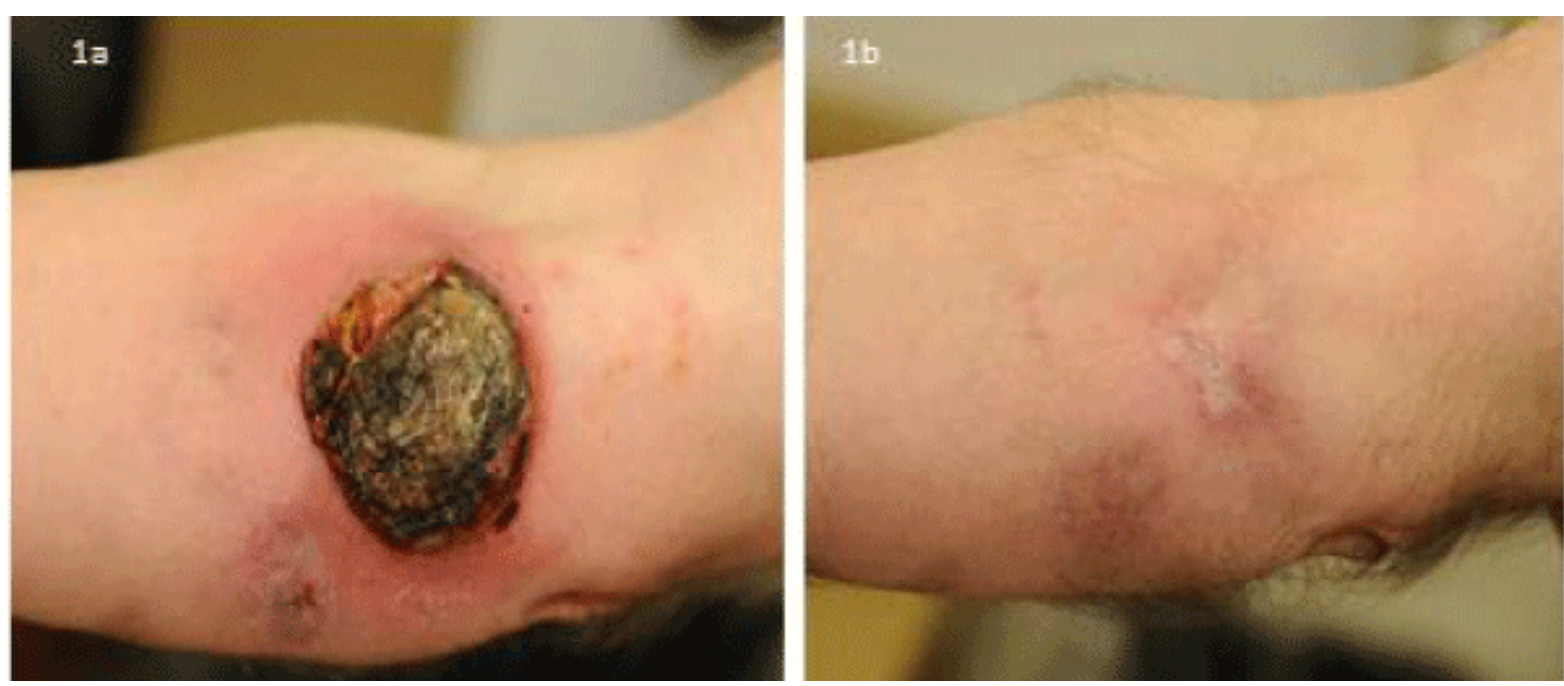

Figure 1: a) Large cell transformation of mycoses fungoides. b) Following Brentuximab vedotin treatment.
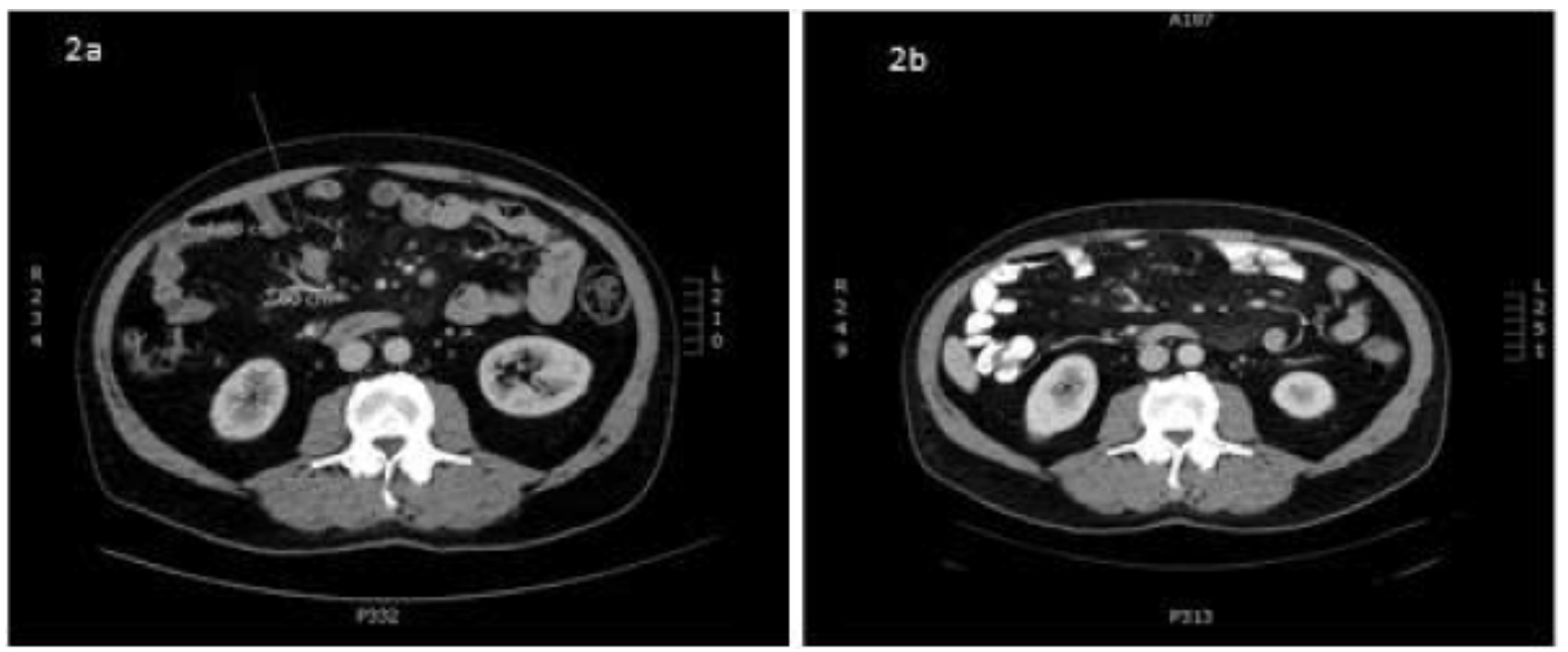

Figure 2: a: Follicular lymphoma pre-rituximab and b: post-rituximab.

\section{Discussion}

Cutaneous T-cell lymphomas (CTCL) are a group of Non-Hodgkin Lymphomas that primarily involve the skin and may over time progress to involve lymph nodes or visceral organs. MF is the most common type of CTCL. The mainstay of therapy in early MF is to gain control of cutaneous symptoms with topical therapies. Therefore, in patients with early limited disease, the initial treatment consists of skin directed treatments such as corticosteroids, carmustine, topical retinoids or local radiation. In patients with wide spread involvement, phototherapy such as PUVA and total skin electron beam therapy (TSEBT) is recommended [1].

Radiation therapy is reported to be the single most effective treatment of MF however; most patients develop recurrent disease over time. TSEBT specifically has been shown to be the most effective form of radiation therapy for MF [2]. Over time, TSEBT doses have increased from 8Gy to $36 \mathrm{~Gy}$ (delivered over 6-10 weeks) due to improved complete response rates and advances in symptom management to improve tolerability. Toxicities such as dermatitis, alopecia and anhidrosis are associated with increased radiation doses. A second course of TSEBT may be considered at relapse for patients that have shown a durable response to the initial course. Low-dose TSEBT (12Gy) has been shown to provide reliable and rapid reduction of MF lesions with less toxicity; but is only effective as a measure to decrease disease burden [3]. Patients who do not respond to SDT are considered for systemic chemotherapy.

Large cell transformation (LCT) of MF is diagnosed when large cells are present in more than $25 \%$ of the tumor infiltrate in the skin biopsy. The prognosis of LCT is usually poor with a median overall survival of 19-36 months. This transformation is rare in early stage disease with a higher incidence of approximately $55 \%$ in stage IV MF. Extra cutaneous transformation carries a worse prognosis with a 5 year 
actuarial survival of $7.8 \%$ [4]. CD30 expression is commonly seen in LCT and MF, therefore anti-CD30 directed therapy, such as BV could play a major role in the treatment of LCT or MF [5,6]. BV is FDA approved for the treatment of relapsed Hodgkin lymphoma and systemic anaplastic large-cell lymphoma.

The case presented suggests promising use of $\mathrm{BV}$ to achieve remission in a patient with CD30+ LCT. There have been reports of BV demonstrating great efficacy in refractory MF but to our knowledge this is the first case report of successful use of BV for LCT of MF. Future studies are needed to help define the efficacy and duration of $\mathrm{BV}$ in patients with LCT and other CD30+ lymphoproliferative disorders.

In this study, we highlight the unusual occurrence of a concurrent diagnosis of follicular B-cell lymphoma in a patient with LCT. CTCL such as MF has been described in association with secondary malignancies. These malignancies may present, simultaneously or subsequent to the diagnosis of CTCL. Overall, the relative frequency of secondary malignancies in CTCL is about $15.6 \%$ [7]. However, the coexistence of CTCL and B-cell malignancies is rare. Most descriptions are isolated case reports and case series [8,9]. Unusual presentations of MF following a diagnosis of idiopathic CD4+ lymphocytopenia or involvement of the GI tract have been reported $[10,11]$.

It is speculated that malignant T-cells promote B-cell hyperproliferation leading to B-cell lymphoma. T-lymphocytes play a large role in the activation and maturation of secretory B-cells through release of immune mediators that bind to B-cell receptors resulting in proliferation and differentiation. CTCL more often precedes a second B-cell lymphoproliferative disorder suggesting that malignant helper/ inducer T-cells may play a large role in immune surveillance mechanisms and altering the cytokine milieu that may modulate the Bcell system resulting in malignancy [8].

There is speculation that modifications prior to the development of the $\mathrm{B}$ and T-cell lineages result in predisposition to the formation of both subtypes of Non-hodgkin lymphoma. This theory centers on a pleuripotent stem cell with the potential to generate both malignant monoclonal $\mathrm{T}$ and $\mathrm{B}$ cells. Evidence of this has been documented in reports of lymphoma with multiple clones of different B and T-cell lineages coexisting in the same tissue [12]. In our patient, MF and FL presented in a sequential pattern and were isolated in different tissues. This suggests a possible subsequent, acquired alteration to the inherent pluripotent stem cell resulting in an oncogenic activation.

In conclusion, the presence of adenopathy in patients with known MF requires biopsy confirmation as the possibility of a second malignancy may be as high as $16 \%$. Our patient had a durable response with monoclonal antibody therapy despite a short duration of initial treatment as compared with his prior therapies. The optimal duration and combination of monoclonal antibody therapy with chemotherapy is an area of ongoing research.

\section{References}

1. Zelenetz AD, Gordon LI, Wierda WG, Abramson JS, Advani RH, et al. (2014) Non-Hodgkin's lymphomas, version 4.2014. Journal of the National Comprehensive Cancer Network: JNCCN 12: 1282-1303.

2. Hoppe RT (2003) Mycosis fungoides: radiation therapy. Dermatol Ther 16: 347-354.

3. Moraes FY, Carvalho Hde A, Hanna SA, Silva JL, Marta GN (2014) Literature review of clinical results of total skin electron irradiation (TSEBT) of mycosis fungoides in adults. Reports of practical oncology and radiotherapy: journal of Greatpoland Cancer Center in Poznan and Polish Society of Radiation Oncology 19: 92-98.

4. Salhany KE, Cousar JB, Greer JP, Casey TT, Fields JP, et al. (1988) Transformation of cutaneous $\mathrm{T}$ cell lymphoma to large cell lymphoma. A clinicopathologic and immunologic study. Am J Pathol 132: 265-277.

5. Reddi DM, Sebastian S, Wang E (2013) Acquisition of CD30 and CD15 Accompanied With Simultaneous Loss of All Pan-T-Cell Antigens in a Case of Histological Transformation of Mycosis Fungoides With Involvement of Regional Lymph Node: An Immunophenotypic Alteration Resembling Classical Hodgkin Lymphoma. The American Journal of dermatopathology 37: 249-253.

6. Forero-Torres A, Bartlett NL, Berryman RB, Chen R, Matous JV, et al. (2014) Extended treatment with brentuximab vedotin in patients with relapsed or refractory CD30-positive hematological malignancies. Leukemia \& lymphoma 7: 1-3.

7. Olsen EA, Delzell E, Jegasothy BV (1984) Second malignancies in cutaneous T cell lymphoma. J Am Acad Dermatol 10: 197-204.

8. Allué L, Domingo A, Moreno A, Crespo N, Marcoval J, et al. (1990) Simultaneous occurrence of cutaneous $\mathrm{T}$ cell lymphoma and low-grade B cell lymphoproliferative diseases. A report of two cases. J Am Acad Dermatol 23: 677-681.

9. Barzilai A, Trau H, David M, Feinmesser M, Bergman R, et al. (2006) Mycosis fungoides associated with B-cell malignancies. Br J Dermatol 155: 379-386.

10. Piccin A, Eisendle K, Rovigatti U, Steurer M, Tauber M, et al. (2014) Transition of idiopathic CD4 + lymphocytopenia into mycosis fungoides? Leuk Lymphoma 55: 1649-1651.

11. Velagapudi P, Turagam M, Uzoaru I, Graham D (2011) Small bowel obstruction due to mycosis fungoides: an unusual presentation. Am J Med Sci 341: 508-509.

12. Gniadecki R (2004) Neoplastic stem cells in cutaneous lymphomas: evidence and clinical implications. Arch Dermatol 140: 1156-1160. 